\title{
PROJETO PRELIMINAR DE UMA UNIDADE PRODUTORA DE UREIA A PARTIR DE TÉCNICAS DE SIMULAÇÃO E OTIMIZAÇÃO DE PROCESSOS
}

\author{
C. A. G. PERLINGEIRO ${ }^{1}$, M. M. V. M SOUZA ${ }^{2}$, R. B. M. $\operatorname{COSTA}^{3}$ e T. F. R. A. RIBEIRO ${ }^{3}$ \\ ${ }^{1}$ Universidade Federal do Rio de Janeiro/Escola de Química, Departamento de Engenharia Química \\ ${ }^{2}$ Universidade Federal do Rio de Janeiro/Escola de Química, Departamento de Processos Inorgânicos \\ ${ }^{3}$ Universidade Federal do Rio de Janeiro/Escola de Química \\ E-mail para contato: ribeiro.tfr@gmail.com
}

\begin{abstract}
RESUMO - O presente estudo consiste na aplicação de técnicas de simulação e otimização de processos para determinação do potencial econômico de uma nova unidade de produção de ureia. A partir de uma prévia revisão econômica e tecnológica, foi possível a definição do fluxograma embrião e, consequentemente, a listagem dos equipamentos e respectivas modelagens matemáticas (Balanços Materiais e Energéticos). Caracterizou-se o problema como sendo de otimização, com uma sequência de cálculo cíclica. Para resolução do sistema foi empregado o método Hooke \& Jeeves de otimização, visando à maximização do lucro do empreendimento, a partir de uma função objetivo desenvolvida através dos critérios do método Venture Profit. Os resultados obtidos da análise indicaram um cenário positivo para implementação da unidade proposta. Por fim, foi conduzida uma análise de sensibilidade em relação às variáveis desconhecidas para determinação da incerteza dos parâmetros empregados.
\end{abstract}

\section{INTRODUÇÃO}

A ureia é um componente nitrogenado de fórmula química $\mathrm{CO}\left(\mathrm{NH}_{2}\right)_{2}$, que desempenha um importante papel na balança comercial brasileira e no setor agropecuário por sua vasta utilização como fertilizante. Atualmente os únicos produtores de ureiano Brasil são a Vale Fertilizantes e a Petrobras, caracterizando um duopólio que responde pela produção de 1,5 milhões de toneladas por ano. Contudo, estima-se que a demanda brasileira ultrapassa a atual média de crescimento mundial de $3 \%$ a.a estimada por Meessen (2012). Este índice é acelerado pelo aumento da demanda de produção de alimentos para exportação e consumo nacional, oriundas do crescimento populacional. Logo, caracteriza-se como motivação de pesquisa a quantificação da receptividade do mercado para inserção de uma nova unidade produtora de ureia e o consequente estudo da viabilidade tecnológica de tal empreendimento, sendo este último o foco do presente estudo.

A partir das projeções econômicas estimadas por Costa e Ribeiro (2014), são aplicados os conceitos da Engenharia de Processos propostos por Perlingeiro (2005) para identificação do potencial econômico da nova unidade. 


\section{9 a 22 de outubro de 2014 \\ Florianópolis/SC}

\section{TECNOLOGIA DE PRODUÇÃO DE UREIA}

O ponto inicial na produção industrial de ureia é a síntese de Basaroff, na qual a ureia é obtida pela desidratação do carbamato de amônio a alta temperatura e pressão. Esta reação é constituída de duas etapas, conforme a Equação 1 e Equação 2:

$2 \mathrm{NH}_{3}+\mathrm{CO}_{2} \leftrightarrow \mathrm{NH}_{2} \mathrm{CO}_{2} \mathrm{NH}_{4}$

$\mathrm{NH}_{2} \mathrm{CO}_{2} \mathrm{NH}_{4} \leftrightarrow \mathrm{H}_{2} \mathrm{O}+\mathrm{CO}\left(\mathrm{NH}_{2}\right)_{2}$

A primeira reação, de formação do carbamato de amônio, é exotérmica $(\Delta \mathrm{H}=-117 \mathrm{~kJ} / \mathrm{mol})$ e muito rápida em ambas as direções, de tal maneira que pode ser considerada em equilíbrio nas condições industriais. A segunda reação, de desidratação do carbamato de amônio, é endotérmica $(\Delta \mathrm{H}$ $=+15,5 \mathrm{~kJ} / \mathrm{mol})$, não alcança o equilíbrio e é a etapa lenta, favorecida com o aumento da temperatura. Como é produzido mais calor na primeira reação do que na segunda, o processo global é exotérmico.

Pesquisadores como Inoue (1972) e Meesen (2012) assumem existir um equilíbrio líquidovapor e que amônia e o dióxido de carbono reagem na fase líquida. Trata-se de um equilíbrio descrito como altamente complexo que envolve reações químicas, formação de azeótropos e estados supercríticos. Logo, este mecanismo ainda não é bem determinado, sendo alvo de extensos estudos. Desde os primeiros modelos desenvolvidos por Frejacquès, que não levavam em conta a formação do intermediário carbamato, diversos novos equacionamentos foram propostos, dentre os mais consagrados os de Inoue (1972), Irazoqui (1993), Isla (1993) e Hamidipour (2005). Mesmo apresentando diferentes origens, a maioria dos modelos compartilham alguns pontos em comum que evidenciam peculiaridades e limitações cinéticas: o aumento da concentração de água diminui a conversão global do processo; a conversão dos reagentes em ureia em função da temperatura passa por um ponto de máximo; a razão molar de $\mathrm{NH}_{3} / \mathrm{CO}_{2}$ acima das proporções estequiométricas favorece a conversão global do sistema. Desde a implementação das primeiras unidades de ureia, esses fatores foram levados em maior ou menor importância para obtenção de altas conversões. Dentre as principais tecnologias aplicadas para produção do fertilizante, pode-se citar: Processo com Única Passagem pelo Reator, Processo de Reciclo Parcial, Processo Convencional, Processo via Stripping Térmico, Processos via Stripping de Amônia e Processos via Stripping de Dióxido de Carbono.

\section{METODOLOGIA}

A primeira etapa para determinação da viabilidade tecnológica de uma nova unidade consiste em uma análise macroeconômica sobre o produto a ser comercializado. Os autores Costa e Ribeiro (2014) evidenciaram em um prévio estudo que o potencial preliminar para implementação de uma nova unidade, também conhecido como Margem Bruta, é considerável e aponta a favor de um novo empreendimento. Naquele trabalho, foram empregados os métodos de regressão e extrapolação de dados para determinação dos parâmetros econômicos necessários para a caracterização da viabilidade da unidade em um determinado ano base, que arbitrariamente foi escolhido em 2025. Estes dados podem ser resumidos na Tabela 1 . 
Tabela 1 - Dados Econômicos para 2025

\begin{tabular}{|c|c|c|}
\hline Dado & Valor & Unidade \\
\hline Preço NH3 & 13,11 & US\$/mol \\
\hline Preço CO2 & 9,52 & US\$/mol \\
\hline Preço H2O & 0,13 & US\$/mol \\
\hline Preço Ureia & 326,13 & US\$/mol \\
\hline Margem Bruta & 290,51 & US\$ \\
\hline Meta de Produção & 2120500,00 & ton $/$ ano \\
\hline
\end{tabular}

Dado que o potencial econômico de uma unidade depende de diversos fatores técnicos, é necessário definir o tipo de processo a ser empregado, uma vez que diferentes tecnologias apresentam distintas listagens de equipamentos, consumo de utilidades e eficiência. Optou-se por utilizar o Processo Stripping via Dióxido de Carbono pelos motivos reportados em Yan (1998) e Meensen (2012): melhores conversões dos reagentes do que as de reciclo parcial; unidades são mais flexíveis; possibilidade de integração energética; tecnologia mais difundida globalmente; alta confiabilidade e segurança; menores contaminações nas correntes a jusante do que na tecnologia de Stripping via $\mathrm{NH}_{3}$. Logo, definida a escolha da tecnologia a ser empregada, é possível reduzir o sistema original ao conjunto de quatro blocos esquematizados no Fluxograma Embrião, representado pela Figura 1.

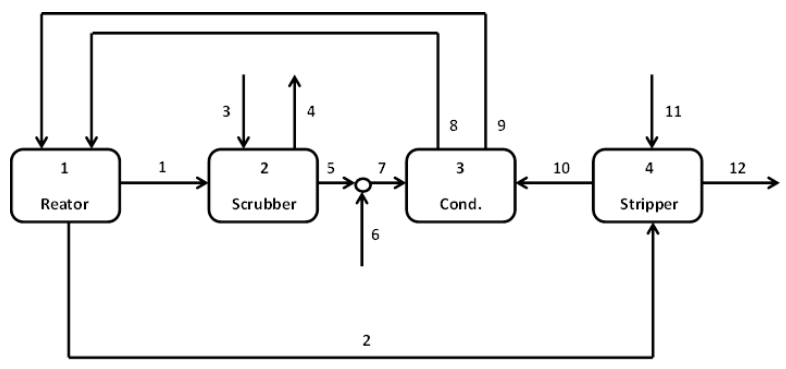

Figura 1. Fluxograma Embrião.

Através das validações propostas por Hamidipour (2005), foram empregadas três simplificações para modelagem dos equipamentos correspondentes aos blocos: todos os equipamentos da região de alta pressão que apresentam reação química podem ser modelados como reator CSTR; a cinética de formação do carbamato é proporcional à utilidade consumida pelo equipamento, quando este está associado a um trocador de calor; toda a amônia livre no líquido e/ou gerada pela decomposição do carbamato são carreadas pelo fluido de stripping, ou seja, o dióxido de carbono.

\subsection{Equacionamento}

Conforme proposto por Perlingeiro (2005), foi empregado o modelo estacionário para previsão da viabilidade técnica e comercial do empreendimento. Logo, assumindo-se a utilização de reatores do tipo CSTR, os balanços de massa e energia assumem a forma da Equação 3 e Equação 4:

$$
\left(\sum_{i, j} f i, j\right)_{\text {entra }}-\left(\sum_{i, j} f i, j\right)_{\text {sai }}+\sum_{k, l}\left(\mp R_{k}\right) * V_{l}=0
$$


$\left.\left(\sum_{i, j} f i, j * C p_{i} * \Delta T\right)_{\text {entra }}-\left(\sum_{i, j} f i, j * C p_{i} * \Delta T\right)\right)_{s a i}+\left(\sum_{k, l}\left(\mp R_{k}\right) * V_{l} * \Delta H_{k}\right) \mp Q_{l}=0$

Para simplificação dos cálculos e minimização do esforço computacional, não foi levado em conta a presença de inertes e subprodutos nesta modelagem, incluindo apenas 5 (cinco) componentes: amônia, dióxido de carbono, carbamato, água e ureia. Para organização da modelagem, prosseguiu-se com o detalhamento do fluxograma do processo de Stripping via $\mathrm{CO}_{2}$ através da inclusão dos trocadores de calor a numeração de cada corrente no processo, conforme a Figura 2:

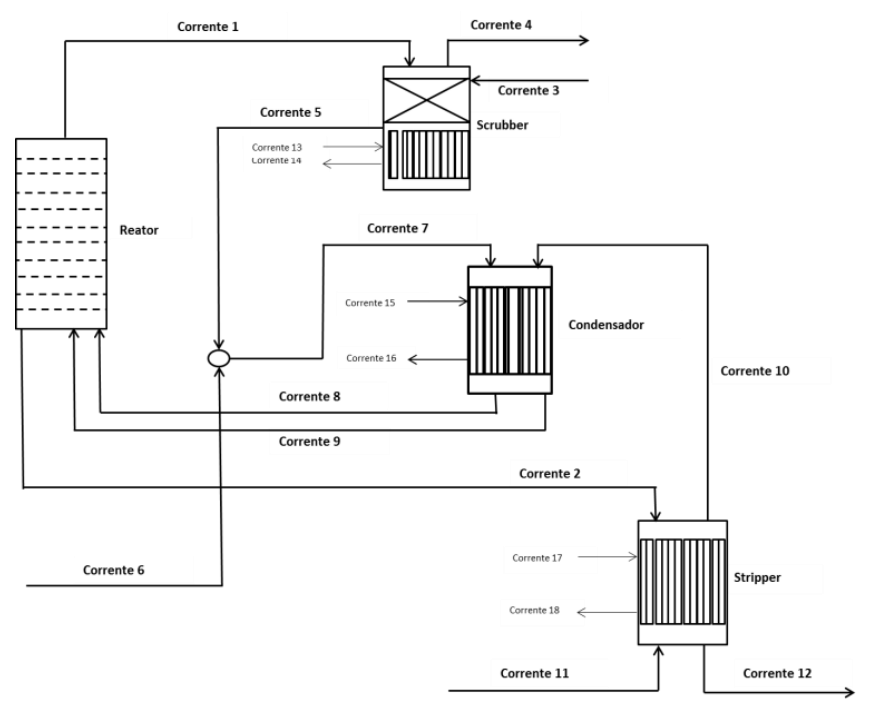

Figura 2. Fluxograma de Processo da Região de Alta Pressão.

Para a modelagem cinética das reações de formação e desidratação do carbamato, foram empregadas as expressões de Egane Claudel, conforme a Equação 5 e Equação 6, respectivamente:

$$
\begin{aligned}
& \log K_{p}=-\frac{7.6569 * 10^{3}}{T}+22.161 \\
& \frac{d x}{d r}=k(1-x) *(a+x)
\end{aligned}
$$

Optou-se por estes modelos dada a simplicidade e confiabilidade reportados por Santos e Oliveira (2010) e Hamidipour (2005). A Equação 5 leva em conta a hipótese de que a reação de formação do carbamato é exotérmica e atinge o equilíbrio, podendo ter seu equilíbrio calculado rapidamente por $K_{p}$.Contudo, a equação de desidratação do carbamato é lenta e endotérmica. Apesar dos estudos de Irazoqui (1993) e Isla (1993) apontarem que a cinética de formação de ureia é homogênea e ocorre na fase líquida, Hamidipour (2005) propôs a utilização de um modelo heterogêneo, que apresenta fácil implementação numérica e resultados satisfatórios.

\subsection{Estratégia de Cálculo}

O modelo proposto retornou um sistema de 86 equações e 122 variáveis. Destas variáveis, deve- 
se descontar as condições conhecidas e metas de projeto, conforme a Tabela 2.

Tabela 2 - Variáveis especificadas: condições conhecidas e metas de projeto.

\begin{tabular}{|c|c|c|c|}
\hline Área Global de Transferência dos Trocadores & $580 \mathrm{~W} / \mathrm{m}{ }^{2} . \mathrm{K}$ & Temperatura da Corrente 1 & $185^{\circ} \mathrm{C}$ \\
\hline Calor Latente De vaporização da água & $41996,8 \mathrm{~kJ} / \mathrm{kg}$ & Temperatura da Corrente 3 & $74{ }^{\circ} \mathrm{C}$ \\
\hline Capacidade Calorífica a Pressão Constante de NH3 & $41,5 \mathrm{~J} / \mathrm{mol} . \mathrm{K}$ & Temperatura da Corrente 4 & $102^{\circ} \mathrm{C}$ \\
\hline Capacidade Calorífica a Pressão Constante de CO2 & $44,1 \mathrm{~J} / \mathrm{mol} . \mathrm{K}$ & Temperatura da Corrente 5 & $165{ }^{\circ} \mathrm{C}$ \\
\hline Capacidade Calorífica a Pressão Constante do Carbamato & $121 \mathrm{~J} / \mathrm{mol} . \mathrm{K}$ & Temperatura da Corrente 6 & $49^{\circ} \mathrm{C}$ \\
\hline Capacidade Calorífica a Pressão Constante de H20 & $81,8 \mathrm{~J} / \mathrm{mol} . \mathrm{K}$ & Temperatura da Corrente 7 & $123^{\circ} \mathrm{C}$ \\
\hline Capacidade Calorífica a Pressão Constante de Ureia & $342,2 \mathrm{~J} / \mathrm{mol} . \mathrm{K}$ & Temperatura da Corrente 8 & $179{ }^{\circ} \mathrm{C}$ \\
\hline Entalpia da Equação 1 & $-117 \mathrm{~kJ} / \mathrm{mol}$ & Temperatura da Corrente 11 & $120^{\circ} \mathrm{C}$ \\
\hline Entalpia da Equação 2 & $15,5 \mathrm{~kJ} / \mathrm{mol}$ & Temperatura da Corrente 12 & $172{ }^{\circ} \mathrm{C}$ \\
\hline Energia de Ativação & $42000 \mathrm{~kJ} / \mathrm{kg} \cdot \mathrm{mol}$ & Temperatura da Corrente 16 & $120^{\circ} \mathrm{C}$ \\
\hline Constante pré-exponencial da Equação 2 & 190000 & Temperatura da Corrente 14 & $120^{\circ} \mathrm{C}$ \\
\hline Constante dos dases Perfeitos & $82,05 \mathrm{E}-6 \mathrm{~m} \mathrm{~m}^{3} \cdot \mathrm{atm} /(\mathrm{K} \cdot \mathrm{mol})$ & Temperatura de Entrada do Vapor & $209^{\circ} \mathrm{C}$ \\
\hline Razão Inicial de água no Reator & $5,9 \mathrm{kmol} / \mathrm{m}^{3}$ & Temperatura de Entrada de Água & $80^{\circ} \mathrm{C}$ \\
\hline Razão Inicial de água no Stripper & $3,8 \mathrm{kmol} / \mathrm{m}^{3}$ & $\mathrm{f} 4,12$ - Meta de Produção de Ureia & $6700 \mathrm{kmol} / \mathrm{h}$ \\
\hline
\end{tabular}

Logo, o sistema a ser analisado apresentou seis graus de liberdade. Isso significa que o problema é consistente, porém indeterminado e para sua resolução deve-se aplicar um método de otimização de processos. Com exceção da dimensão do Condensador, cujo resultado é diretamente determinado pelo conjunto de variáveis disponíveis no sistema, os volumes dos demais equipamentos e áreas dos trocadores de calor envolvidos foram escolhidos como variáveis de projeto. Dessa forma, percebe-se que o problema pode ser caracterizado como dimensionamento com otimização. Dentre as diferentes abordagens possíveis para resolução do sistema, foi empregado o método sequencial de resolução, também conhecido como "Equation Oriented”. Esta metodologia resolve as equações sucessivamente, transmitindo os valores das variáveis de uma equação para outra. Através do Algoritmo de Ordenação de Equações (AOE) indicado por Perlingeiro (2005), notou-se o aparecimento de ciclos, que são estruturas cuja uma determinada variável depende de si mesma para ser calculada. Para resolução desses subsistemas, empregou-se o Método da Bisseção.

Foram manipuladas as 06 (seis) dimensões dos equipamentos, na busca da solução ótima do problema, cujo critério será definido como o maior Lucro do Empreendimento. Para construção desta equação, foi empregado o método Venture Profit descrito por Perlingeiro, (2005), levando em conta dados como: dimensão dos equipamentos, Custo Fixo, Custo de Matéria Prima, Custo de Utilidades e etc. Logo, a função objetivo assumiu a forma da Equação 7:

$$
\begin{aligned}
& L E=0,6762 \times R-0,959 \times(\text { Cmatprim }+ \text { Cutil })-62720,35 \times(V 1+V 2+V 3+V 4)^{0,53}- \\
& 6043,181333 \times(S 1+S 2+S 3)^{0,59}
\end{aligned}
$$

Diferentes métodos, dentre os analíticos e numéricos, poderiam ser utilizados para executar a otimização de funções multivariáveis. Optou-se pelo método Hooke \& Jeeves, por sua simplicidade, eficiência e robustez. Este método se baseia na seleção de um ponto-base e o cálculo do valor da Função Objetivo neste ponto. É conduzido um conjunto de explorações e progressões até o atingimento do ótimo que se encontre dentro das tolerâncias estabelecidas. 


\section{RESULTADOS E DISCUSSÕES}

O programa desenvolvido em EXCEL-VBA convergiu em aproximadamente 80 iterações para retornar o conjunto de variáveis que atingisse o critério de ótimo.A fim de se analisar a consistência da modelagem proposta, optou-se pela comparação da fração molar da corrente de saída do Stripping com os dados divulgados por Santos e Oliveira (2010) em estudo realizado com o software de processo CHEMCAD, conforme a Tabela 3:

Tabela 3 - Resultados Calculados da Corrente de Ureia para Concentração

\begin{tabular}{|c|c|c|c|}
\hline Corrente 12 & Valor & \% Calculado & $\%$ Santos e Costa \\
\hline W12 $(\mathrm{kmol} / \mathrm{h})$ & 1614657,21 & - & - \\
\hline $\mathrm{f} 112(\mathrm{kmol} / \mathrm{h})$ & 319843,75 & $19,81 \%$ & $14,80 \%$ \\
\hline $\mathrm{f} 212(\mathrm{kmol} / \mathrm{h})$ & 199902,34 & $12,38 \%$ & $7,39 \%$ \\
\hline $\mathrm{f} 312(\mathrm{kmol} / \mathrm{h})$ & 211,12 & $0,01 \%$ & - \\
\hline $\mathrm{f} 412(\mathrm{kmol} / \mathrm{h})$ & 410000 & $25,39 \%$ & $29,74 \%$ \\
\hline $\mathrm{f} 512(\mathrm{kmol} / \mathrm{h})$ & 684700 & $42,41 \%$ & $48,07 \%$ \\
\hline
\end{tabular}

Apesar de simular ambientes e metas diferentes, ambos os trabalhos empregaram a mesma tecnologia, ou seja, utilizaram o conceito de stripping via dióxido de carbono. Ao contrário da vazão molar, que depende das metas de produção, a fração molar pode indicar a validade do modelo, já que unidades similares tendem a apresentar semelhança composicional. Pode-se concluir então que os resultados calculados apresentam tendências semelhantes aos reportados por esta referência, exprimindo um maior distanciamento na corrente de $\mathrm{CO}_{2}$. Esse efeito provavelmente ocorre uma vez que no presente estudo foi levado em conta a formação do carbamato de amônio, enquanto que naquele trabalho este intermediário foi negligenciado. Como a cinética empregada por Santos e Costa (2010) assumia a conversão de $\mathrm{CO}_{2}$ diretamente em ureia, ou seja, negligenciando a reação de desidratação que é a etapa limitante do processo, é de ser esperar que tal modelo superestime o consumo deste reagente, assim como a formação de ureia.

Por sua vez, os parâmetros econômicos avaliados indicaram um cenário positivo para o investimento neste empreendimento, conforme apontado pela Tabela 4.

Tabela 4 - Resultados Econômicos

\begin{tabular}{|c|c|}
\hline Meta de Produção (ton/ano) & 2120500,00 \\
\hline Preço ureia (US\$/kg) & 5,43 \\
\hline Receita (\$/ano) & 39846957,12 \\
\hline Custo Mat. Prima (\$/ano) & 12379805,05 \\
\hline Custo Utilidade (\$/ano) & 14398,00 \\
\hline Lucro do Empreendimento (\$/ano) & 24386987,48 \\
\hline
\end{tabular}

Além de um expressivo lucro, a unidade proposta apresentou uma baixa estimativa de investimento total, na ordem de 3 (três) milhões de dólares. Associado ao planejamento estratégico de crescimento no setor agropecuário e a necessidade de melhoria da balança comercial, estes índices justificariam que houvesse novos fomentos de capital público e/ou privado para o prosseguimento com a construção de tal unidade. Contudo, vale ressaltar que esses índices foram obtidos baseados em 


\section{9 a 22 de outubro de 2014 \\ Florianópolis/SC}

empreendimentos acoplados a unidades produtoras de amônia, tais como ocorre na Vale Fertilizantes e Petrobras. A inserção de uma nova unidade fisicamente distante de uma unidade produtora de amônia ou por uma empresa terceira, obrigatoriamente apresentaria redução do potencial econômico, uma vez que os reagentes apresentam alta participação no Lucro do Empreendimento.

Notou-se também que a participação do custo da matéria prima é muito superior ao custo de utilidade da unidade. Logo, é possível concluir que esta unidade de fertilizante é altamente sensível aos impactos e/ou flutuações de mercado que os gases de síntese podem sofrer. Uma vez que ambos os reagentes estão associados a rotas petroquímicas, espera-se que empresas de exploração e produção de petróleo, tais como a Petrobras, apresentem maior confiabilidade em seus processos.

As variáveis manipuladas pelo algoritmo de otimização, ou seja, as dimensões dos reatores CSTR e os trocadores de calor forneceram os valores da Tabela 5. Evidenciou-se durante a rodada que o algoritmo tendeu a minimizar no possível o volume dos equipamentos, pois fixada a meta de produção, essa redução de valores minimiza o consumo e custo de matéria prima, que desempenha importante papel na função objetivo. Notou-se também que por terem sido empregados modelos idênticos de reator CSTR e trocador para os equipamentos no cálculo da função objetivo, o algoritmo retornou os mesmos valores para cada equipamento. Isso aponta que a hipótese proposta por Hamidipour (2005) pode não ser a mais indicada para este tipo de estudo, uma vez que não são observadas as contribuições individuais de cada equipamento. Contudo, para propósitos de detalhamento técnico das correntes do fluxograma e estimativa preliminar do potencial do empreendimento, esta simplificação se mostrou válida por permitir um entendimento inicial sobre a natureza do empreendimento.

Tabela 5 - Dimensão dos Equipamentos

\begin{tabular}{|c|c|c|c|c|}
\hline & Reator & Condensador & Scrubber & Stripper \\
\hline Volume do Equipamento $\left(\mathrm{m}^{3}\right)$ & 11,16 & 11,16 & 11,16 & 11,16 \\
\hline Área do Trocador $\left(\mathrm{m}^{2}\right)$ & - & - & 10,06 & 10,06 \\
\hline
\end{tabular}

Por fim, decidiu-se avaliar a extensão do impacto das simplificações adotadas através de uma análise de sensibilidade das variáveis mais incertas do projeto conforme sugerido por Perlingeiro (2005), a fim de se analisar o comportamento dos principais resultados obtidos. Observou-se que os resultados das incertezas se mantiveram abaixo de $1 \%$, assegurando confiabilidade às simplificações tomadas. Os parâmetros que mais sofreram influência foram o coeficiente global de transferência de calor empregado como meta dos trocadores de calor e a razão de entrada ótima dos reagentes $\mathrm{NH}_{3} \mathrm{e}$ $\mathrm{CO}_{2}$, sugerindo adotá-los como possíveis variáveis manipuladas em futuros projetos.

\section{CONCLUSÕES}

O presente estudo teve como objetivo avaliar a viabilidade tecnológica de uma nova unidade produtora de ureia no mercado nacional, dado as crescentes tendências de aumento de consumo deste produto. A partir da definição da meta de produção e processo a ser empregado, foi possível a definição do fluxograma embrião e listagem dos equipamentos a serem adotados na região de síntese (alta pressão). A partir da análise do balanço de informações do sistema de equações oriunda dos 
balanços materiais e energéticos, concluiu-se que se trata de um problema dedimensionamento com otimização com presença de ciclos. O critério de otimização empregado foi o de maximização da função lucro, que foi obtida através do método Venture Profit.

A modelagem proposta apresentou resultados coerentes com os dados da literatura. Contudo, alguns desvios puderam ser observados, provavelmente oriundos do emprego de diferentes modelos cinéticos. Os parâmetros econômicos apontaram um cenário positivo para implementação da unidade, com expressivo lucro e baixo investimento inicial. Observou-se que pela alta participação do custo das matérias primas, empreendimentos acoplados com unidades produtoras de amônia provavelmente apresentam vantagem sobre unidades isoladas. Por fim, observou-se que apesar da simplificação proposta por Hamidipour (2005) de empregar reatores CSTR idênticos ser válida para a especificação técnica das correntes e entendimento inicial sobre a natureza do empreendimento, esta não seria a mais adequada quando associada ao método Venture Profit por não capturar as contribuições individuais de cada equipamento.

Como sugestões para futuros trabalhos, recomenda-se: avaliação do potencial econômico de unidades isoladas frente a acopladas; inclusão da formação de subprodutos na modelagem; utilização de equações cinéticas homogêneas; simulação das regiões de recuperação e finalização de ureia; emprego de modelos mais completos para caracterização dos equipamentos da região de alta pressão.

\section{REFERÊNCIAS}

COSTA, R. B. M; RIBEIRO, T. F. R. Determinação de parâmetros econômicos para simulação e otimização de uma unidade produtora de ureia. Rio de Janeiro, 2014.

HAMIDIPOUR, M.;MOSTOUFI, N.; SOTUDEH-GHAREBAGH, R. Modeling the synthesis section of an industrial urea plant. Chem. Eng. J., v. 106, p. 249-260, 2005.

INOUE, S.; KANAI, K.; OTSUKA, E. Equilibrium of Urea Synthesis. Bulletin of the chemical society of Japan, v. 45, p. 1339-1345, 1972.

IRAZOQUI, H.A.; ISLA, M. A.; GENOUD, C.M. Simulation of an urea synthesis reator. 2 Reactor Model. Ind. Eng. Chem. Res., v. 32, p. 2670-2680, 1993.

ISLA, M. A.; IRAZOQUI, H.A.; GENOUD, C.M. Simulation of an urea synthesis reator. 1 Thermodynamic Framework. Ind. Eng. Chem. Res., v. 32, p. 2662-2670, 1993.

MEESSEN, J. H. Urea. Ullmann's Encyclopedia of Industrial Chemistry, v. 37, p. 657-695, 2010.

SANTOS, B. N. dos; OLIVEIRA, D. M. de. Estudo da modelagem e simulação de um processo de produção de ureia. Niterói, 2010.

PERLINGEIRO, C.A.G. Engenharia de Processos. Rio de Janeiro: Edgard Blucher, 2005.

YAN, X. et al. Fertilizer manual. Dordrecht, Editora Kluwer Academic Publishers,1998. 